\title{
Non-interferometric Quantitative Phase Imaging of Yeast Cells
}

\author{
Praveen Kumar Poola, Vimal Prabhu Pandiyan and Renu John* \\ Department of Biomedical Engineering, Indian Institute of Technology Hyderabad, \\ Kandi, Telangana-502285, India. \\ renujohn@iith.ac.in
}

\begin{abstract}
Real-time imaging of live cells is quite difficult without the addition of external contrast agents. Various methods for quantitative phase imaging of living cells have been proposed like digital holographic microscopy and diffraction phase microscopy. In this paper, we report theoretical and experimental results of quantitative phase imaging of live yeast cells with nanometric precision using transport of intensity equations (TIE). We demonstrate nanometric depth sensitivity in imaging live yeast cells using this technique. This technique being noninterferometric, does not need any coherent light sources and images can be captured through a regular bright-field microscope. This real-time imaging technique would deliver the depth or 3-D volume information of cells and is highly promising in real-time digital pathology applications, screening of pathogens and staging of diseases like malaria as it does not need any preprocessing of samples.
\end{abstract}

Keywords: Transport of Intensity Equation (TIE), Noninterferometric microscopy, Quantitative phase imaging

\section{INTRODUCTION}

When a light beam encounters a phase objects such as cells or glass, the light beam does not show any change in it amplitude but these phase samples introduce a phase delay (base on the refractive index differences between the sample and its surrounding) in the light, such a small phase change is neither visible to the human eye nor to the intensity detectors like CCDs or CMOS cameras. Capturing this phase delay is very important as it contains volume information about the sample, including its surface profile, morphology, depth and the refractive index [1] of the sample under investigation. Various techniques have been reported in the past for imaging the phase objects [2-4]. Techniques like Zernike phase contrast microscopy, nomarski differential interference contrast microscopy (DIC) help us in visualization of phase objects by converting phase information into amplitude information through proper mechanisms. But these techniques could help us in providing qualitative data only and failed to deliver quantitative phase information.

Quantitative phase information is an important parameter for biologists in understanding the dynamics of the cells, and is not possible with the existing qualitative techniques, so to overcome the drawbacks of qualitative techniques, researchers around the globe have developed quantitative phase imaging techniques such as Digital holographic microscopy (DHM) [5-6] were developed, which is a quantitative phase imaging (QPI) technique. But the computation load, bulk setup, coherent light source requirements and stringent constraints on interferometric setup for phase stability have plagued this technique in imaging phase objects.

In this paper, we exploit the principle of transport of Intensity equations (TIE) for reconstruction of quantitative phase imaging, which can avoid the problems associated with the DHM technique. The elegance of this technique is that it can be readily implemented with the help of a regular bright-field microscope and its unique noniterative, noninterferometric capability with low-complex optical system makes this technique readily adaptable by biologists. TIE imaging is possible through from axial derivative measurements of captured intensities in the Fresnel region, which are related to the 3-D phase map of the sample under observation. This technique has a good scope for patient bedside diagnosis. The axial intensity derivative can be measured by finite difference method. Teague [7-8] derived TIE for the phase inside of the circularly bounded region by analytically solving a Green's function solution which can be implemented with partially coherent beam as well.

Biophotonics Japan 2015, edited by Takashige Omatsu, Yoshio Hayasaki, Yusuke Ogura, Yasuyuki Ozeki, Seigo Ohno, Proc. of SPIE Vol. 9792, 97920G · C 2015 SPIE · CCC code: 1605-7422/15/\$18 · doi: 10.1117/12.2207232 


\section{METHOD OF PHASE EXTRACTION WITH PARTIALLY COHERENT LIGHT}

The complete complex field can be extracted from Helmholtz equation with paraxial approximation of the wave field. A simple way to obtain the TIE is the use of the parabolic equation [7-8].

$$
\left(i \frac{\partial}{\partial z}+\frac{\Delta_{\perp}}{2 k}+k\right) U=0
$$

Where $\mathrm{k}$ is the wavenumber, $\mathrm{U}$ is the optical field and $\Delta_{\perp}$ is the transverse Laplacian. The wave amplitude $\mathrm{U}$ can be written in terms of intensity and phase as

$$
U(x, y, z)=\sqrt{I(x, y, z)} e^{i \varphi(x, y, z)}
$$

Substituting equation (2) into (1) and taking the imaginary part of the resulting equation produce TIE equation, which relates the first order derivative of axial intensity measurements in the Fresnel region [7], with the phase at the intermediate plane between the object and CCD as given by

$$
\nabla_{\perp} \cdot\left[I\left(r_{\perp}, z\right) \nabla_{\perp} \varphi\left(r_{\perp}, z\right)\right]=-k \frac{\partial I\left(r_{\perp}, z\right)}{\partial z}
$$

where $\nabla_{\perp}$ is transverse two-dimensional gradient operator, $r_{\perp}$ is the position vector representing the transverse spatial coordinates (x,y), and $k=\frac{2 \pi}{\lambda}$ is the propagation constant. The detailed theory of TIE formalism has been explained in cited references and is excluded from this paper. The eqn. (3) relates the through-focus (z axis) intensity measurements to the two dimensional phase distribution and assumes paraxial light propagation approximation and can be inverted to recover the phase [9]. With the assumption of $\vec{\nabla} \varphi=\vec{\nabla} \psi$, a formal solution to eqn. (3) is given by [10]

$$
\varphi\left(r_{\perp}, z\right)=-k \nabla_{\perp}^{-2} \cdot\left\{\nabla_{\perp}\left[\frac{\nabla_{\perp} \nabla_{\perp}^{-2} \partial_{z} I}{I}\right]\right\}, \quad I \neq 0
$$

where $\nabla_{\perp}^{-2}$ is the inverse Laplacian operator. The assumption $\vec{\nabla} \varphi=\vec{\nabla} \psi$ is valid, if $\psi$ (scalar field) is conservative, which means that the path integral around a closed path is zero, and the phase $\varphi$ is single valued and it does not contain any singularities i.e. intensity I is assumed to be strictly positive. The main problem to solve TIE equation is unknown boundary conditions. Which was bypassed by Fast Fourier Transform (FFT) based method by solving the TIE non-iteratively in the frequency domain with implicit periodic boundary conditions. We used a commercial inverted bright-field microscope (Carl Zeiss Axio-observer A1). The illumination is from a halogen lamp having central wavelength of $552 \mathrm{~nm}$. We used microscope objective of $40 \mathrm{x}$ with NA=0.65 for imaging. Fig. 1(a-b) shows the image above focus and below focus images and the defocus of distance $\pm 10 \mu \mathrm{m}$ along the axial direction is considered from the image in focus shown fig. 1(c). TIE from eqn. 4 can be readily applied on the registered images to retrieve phase map/depth profile of the yeast cells. The retrieved 3D profile of the sample is shown in fig. 1(d). Once we have the quantitative phase, we can find the object thickness ' $h$ ' through the below equation as

$$
h=\frac{\phi \lambda}{2 \pi\left(n-n_{\text {medium }}\right)}
$$

Considering refractive index of yeast cells as $(\mathrm{n}=1.49)$ and its medium yeast broth as $\left(\mathrm{n}_{\text {medium }}=1.334\right)$ quantitative thickness of the cells were found to be $1-2 \mu m$ by using eq. 5. A line is drawn on fig. 1(d) marked as 'Line 1' to show the nanometric resolution capability of this technique. From fig. 2. We can see the thickness profile on the yeast cells with nanometric resolution. 


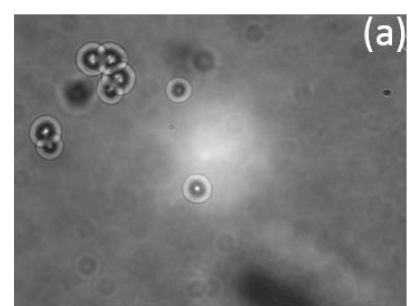

Image above focus

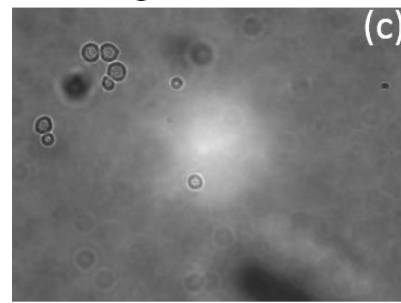

Image in focus

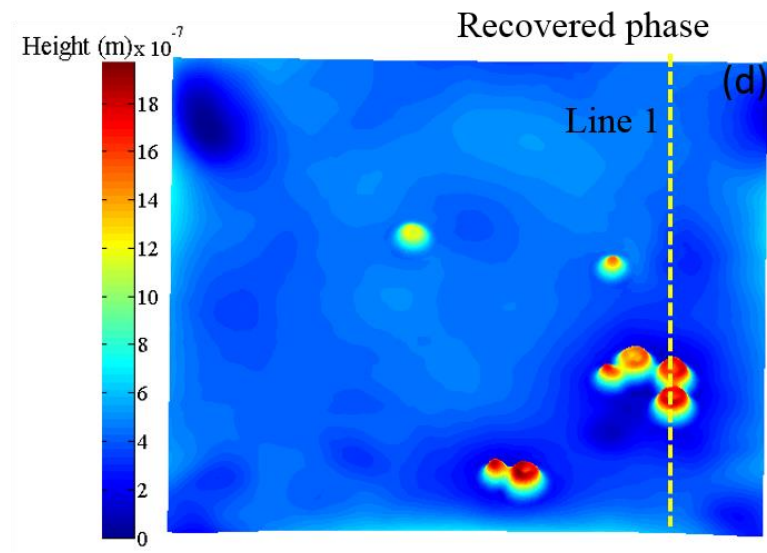

Figure 1. Yeast cells and their 3-D reconstruction (a) Image above focus (b) Image below focus (c) Image in focus (d ) Thickness map with $3 \mathrm{D}$ view

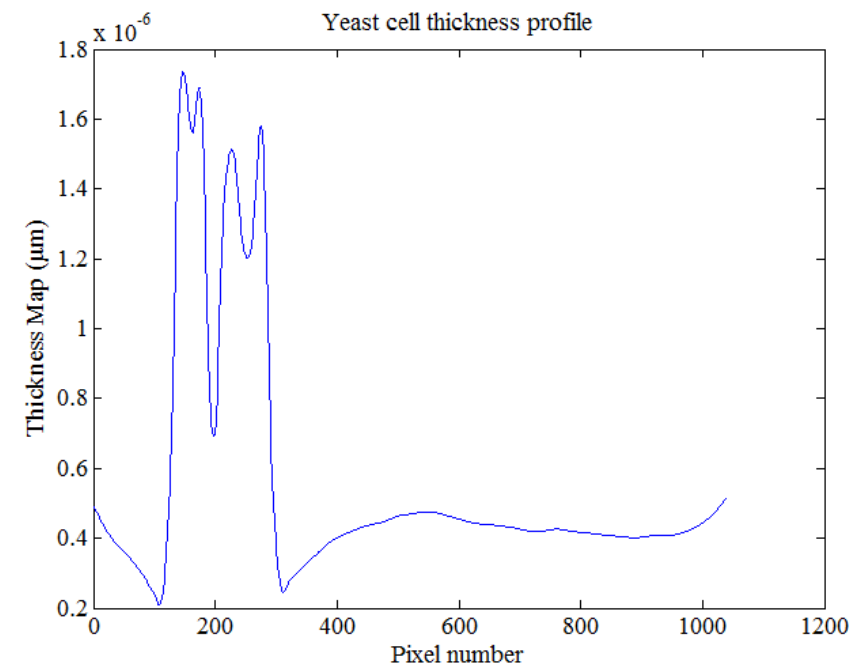

Fig. 2. Yeast cells thickness profile from line 1 in fig. 1(d) 


\section{RESULTS AND DISCUSSION}

We reconstruct the phase of cells noninterferometrically based on the transport of intensity equations (TIE). This technique estimates the axial derivative from positive through-focus intensity measurements. We demonstrate nanometric depth sensitivity in imaging live yeast cells using this technique. Experimental results shown in the paper demonstrate the capability of the technique in 3-D volume estimation of living cells and studying subcellular dynamics in real time. This real-time imaging technique is highly promising in real-time digital pathology applications, screening of pathogens and staging of diseases like malaria as it does not need any preprocessing of samples.

\section{REFERENCES}

[1] Ahmad, D, Shomalia, R.,and Tavassoly, M. T., "Determination of the refractive index profile of a symmetric fiber preform by the transport of intensity equation," Optics \& Laser Technology 40, 850-853 (2008).

[2] Popescu, G., Ikeda, T., Catherine, A.B.,,Badizadegan, K., and Ramachandra, R. D.,"Erythrocyte structure and dynamics quantified by Hilbert phase microscopy," J. Biomed. Opt. 10(6), 060503 (2005).

[3] Yamauchi, T., Iwai, H., Miwa, M. and Yamashita, Y., "Low-coherent quantitative phase microscope for nanometer-scale measurement of living cells morphology," Opt. Express 16, 12227-12238 (2008).

[4] Yamauchi, T., Iwai, H., Miwa, M. and Yamashita, Y., "Lable-free imaging of intracellular motility by low coherent quantitative phase microscopy," Opt. Express 19, 5536-5550 (2011).

[5] Chen, J., Endo, J. and Fujita, H., "Quantitative phase measurement interference microscope for transparent objects", Proc. SPIE 4919, Advanced Materials and Devices for Sensing and Imaging, 364 (2002).

[6] Charrière, F.,Pavillon, N.,Colomb, T., Depeursinge, C.,Heger, J. T.,Edward, A. D.M., Marquet, P., and Rappaz, B., "Living specimen tomography by digital holographic microscopy: morphometry of testate amoeba," Opt. Express 14 (16): 7005-7013 (2006).

[7] Teague, M., "Deterministic phase retrieval: a Green's function solution," J. Opt. Soc. Am.73, 1434-1441 (1983).

[8] M.Teague et al., "Image formation in terms of the transport equation," Journal of the Optical Society of America A, vol.2, no.11, pp.2019-2026, (1985).

[9] Paganin, D. and Nugent, K., "Noninterferometric phase imaging with partially coherent light," Phys. Rev. Lett. 80, 2586-2589 (1998).

[10] Volkov, V. V., Zhu, Y. and DeGraef, M,, "A new symmetrized solution for phase retrieval using the transport of intensity equation," Micron 33 (2002). 